\title{
Block-Transitive 4-(v,k,4) Designs and Ree Groups
}

\author{
Shaojun Dai', Ruihai Zhang² \\ ${ }^{1}$ Department of Mathematics, Tianjin Polytechnic University, Tianjin, China \\ ${ }^{2}$ Department of Mathematics, Tianjin University of Science and Technology, Tianjin, China \\ Email: daishaojun1978@sina.com, zhangruihai781119@126.com
}

Received 27 February 2016; accepted 4 April 2016; published 7 April 2016

Copyright (c) 2016 by authors and Scientific Research Publishing Inc.

This work is licensed under the Creative Commons Attribution International License (CC BY).

http://creativecommons.org/licenses/by/4.0/

(c) () Open Access

\section{Abstract}

This article is a contribution to the study of the automorphism groups of $4-(v, k, \lambda)$ designs. Let $\mathcal{S}=(\mathcal{P}, \mathcal{B})$ be a non-trivial $4-\left(q^{3}+1, k, 4\right)$ design where $q=3^{2 n+1}$ for some positive integer $n \geq 1$, and $G \leq \operatorname{Aut}(\mathcal{S})$ is block-transitive. If the socle of $G$ is isomorphic to the simple groups of lie type ${ }^{2} G_{2}(q)$, then $G$ is not flag-transitive.

\section{Keywords}

\section{Flag-Transitive, Block-Transitive, $t$-Design, Ree Group}

\section{Introduction}

For positive integers $t \leq k \leq v$ and $\lambda$, we define a $t-(v, k, \lambda)$ design to be a finite incidence structure $\mathcal{S}=(\mathcal{P}, \mathcal{B})$, where $\mathcal{P}$ denotes a set of points, $|\mathcal{P}|=v$, and $\mathcal{B}$ a set of blocks, $|\mathcal{B}|=b$, with the properties that each block is incident with $k$ points, and each $t$-subset of $\mathcal{P}$ is incident with $\lambda$ blocks. A flag of $\mathcal{S}$ is an incident point-block pair $(x, B)$ with $x$ is incident with $B$, where $B \in \mathcal{B}$. We consider automorphisms of $\mathcal{S}$ as pairs of permutations on $\mathcal{P}$ and $\mathcal{B}$ which preserve incidence structure. We call a group $G \leq \operatorname{Aut}(\mathcal{S})$ of automorphisms of $\mathcal{S}$ flag-transitive (respectively block-transitive, point $t$-transitive, point $t$-homogeneous) if $G$ acts transitively on the flags (respectively transitively on the blocks, $t$-transitively on the points, $t$-homogeneously on the points) of $\mathcal{S}$. For short, $\mathcal{S}$ is said to be, e.g., flag-transitive if $\mathcal{S}$ admits a flag-transitive group of automorphisms.

For historical reasons, a $t-(v, k, \lambda)$ design with $\lambda=1$ is called a Steiner $t$-design (sometimes this is also known as a Steiner system). If $t<k<v$ holds, then we speak of a non-trivial Steiner $t$-designs. 
Investigating $t$-designs for arbitrary $\lambda$, but large $t$, Cameron and Praeger proved the following result:

Theorem 1. ([1]) Let $\mathcal{S}=(\mathcal{P}, \mathcal{B})$ be a $t-(v, k, \lambda)$ design. If $G \leq A u t(\mathcal{S})$ acts block-transitively on $\mathcal{S}$, then $t \leq 7$, while if $G \leq \operatorname{Aut}(\mathcal{S})$ acts flag-transitively on $\mathcal{S}$, then $t \leq 6$.

Recently, Huber (see [2]) completely classified all flag-transitive Steiner $t$-designs using the classification of the finite 2-transitive permutation groups. Hence the determination of all flag-transitive and block-transitive $t$-designs with $\lambda \geq 2$ has remained of particular interest and has been known as a long-standing and still open problem.

The present paper continues the work of classifying block-transitive $t$-designs. We discuss the block-transitive $4-(v, k, 4)$ designs and Ree groups. We get the following result:

Main Theorem. Let $\mathcal{S}=(\mathcal{P}, \mathcal{B})$ be a non-trivial $4-\left(q^{3}+1, k, 4\right)$ design, where $q=3^{2 n+1}$ for some positive integer $n \geq 1$, and $G \leq A u t(\mathcal{S})$ is block-transitive. If $\operatorname{Soc}(G)$, the socle of $G$, is ${ }^{2} G_{2}(q)$, then $G$ is not flag-transitive.

The second section describes the definitions and contains several preliminary results about flag-transitivity and $t$-designs. In 3 Section, we give the proof of the Main Theorem.

\section{Preliminary Results}

The Ree groups ${ }^{2} G_{2}(q)$ form an infinite family of simple groups of Lie type, and were defined in [3] as subgroups of $G L(7, q)$. Let $G F(q)$ be finite field of $q$ elements, where $q=3^{2 n+1}$ for some positive integer $n \geq 1$ (in particular, $q \geq 27$ ). Let $Q$ is a Sylow 3-subgroup of $G, K$ is a multiplicative group of $G F(q)$ and ${ }^{2} G_{2}(q)$ is a group of order $q^{3}\left(q^{3}+1\right)(q-1)$ (see [4]-[6]). Hence ${ }^{2} G_{2}(q)$ is a group of automorphisms of Steiner $3-\left(q^{3}+1, q+1,1\right)$ design and acts 2-transitive on $q^{3}+1$ points (see [7]).

Here we gather notation which are used throughout this paper. For a $t$-design $\mathcal{S}=(\mathcal{P}, \mathcal{B})$ with $G \leq \operatorname{Aut}(\mathcal{S})$, let $r$ denotes the number of blocks through a given point, $G_{x}$ denotes the stabilizer of a point $x \in \mathcal{P}$ and $G_{B}$ the setwise stabilizer of a block $B \in \mathcal{B}$. We define $G_{x B}=G_{x} \cap G_{B}$. For integers $m$ and $n$, let $(m, n)$ denotes the greatest common divisor of $m$ and $n$, and $m \mid n$ if $m$ divides $n$.

Lemma 1. ([2]) Let $G$ act flag-transitively on $t-(v, k, \lambda)$ design $\mathcal{S}=(\mathcal{P}, \mathcal{B})$. Then $G$ is block-transitive and the following cases hold:

1) $|G|=\left|G_{x}\right|\left|x^{G}\right|=\left|G_{x}\right| v$, where $x \in \mathcal{P}$;

2) $|G|=\left|G_{B}\right|\left|B^{G}\right|=\left|G_{B}\right| b$, where $B \in \mathcal{B}$;

3) $|G|=\left|G_{x B}\right|(x, B)^{G}|=| G_{x B} \mid b k$, where $x \in B$.

Lemma 2. ([8]) Let $\mathcal{S}=(\mathcal{P}, \mathcal{B})$ is a non-trivial $t-(v, k, \lambda)$ design. Then

$$
\lambda(v-t+1) \geq(k-t+2)(k-t+1) .
$$

Lemma 3. ([8]) Let $\mathcal{S}=(\mathcal{P}, \mathcal{B})$ is a non-trivial $4-(v, k, \lambda)$ design. Then

1) $b k=v r$

2) $b=\frac{\lambda v(v-1)(v-2)(v-3)}{k(k-1)(k-2)(k-3)}$.

Corollary 1. Let $\mathcal{S}=(\mathcal{P}, \mathcal{B})$ is a non-trivial $4-(v, k, 4)$ design. If $v=q^{3}+1$, Then $k<3+2 q \sqrt{q}$.

Proof. By Lemma 2, we have $4(v-3) \geq(k-2)(k-3)$. If $v=q^{3}+1$, then

$$
4\left(q^{3}-2\right) \geq(k-2)(k-3) \text {. }
$$

Hence

$$
k^{2}-5 k-4 q^{3}+14 \leq 0 .
$$

We get

$$
k \leq \frac{5+\sqrt{16 q^{3}-31}}{2}<3+2 q \sqrt{q} .
$$




\section{Proof of the Main Theorem}

Suppose that $G$ acts flag-transitively on $4-(v, k, 4)$ design and $v=q^{3}+1$. Then $G$ is block-transitive and point-transitive. Since $T={ }^{2} G_{2}(q) \unlhd G \leq \operatorname{Aut}(T)$, we may assume that $G=T:\langle\alpha\rangle$ and $G=T:(G \bigcap\langle\alpha\rangle)$ by Dedekind's theorem, where $\alpha: x \rightarrow x^{3}, x \in G F(q)$ and $\alpha$ is an automorphism of field $G F(q)$. Let $q=3^{f}, f=2 n+1$ is odd, and $|\langle\alpha\rangle|=m$, then $m \mid f$. Obviously, $|G|=q^{3}\left(q^{3}+1\right)(q-1) m$. $\mathcal{P}$.

First, we will proof that if $g \in G$ fixes three different points of $\mathcal{P}$, then $g$ must fix at least four points in

Suppose that $g \in G,\left|\operatorname{Fix}_{\mathcal{P}}(g)\right| \geq 3, \quad x \in \operatorname{Fix}_{\mathcal{P}}(g)$. Let $P$ is a normal Sylow 3-subgroup of $G_{x}$. Then $\mathcal{P}$ is transitive on $\mathcal{P}-\{x\}$. By $v=q^{3}+1$, we have $|P|=|\mathcal{P}-\{x\}|=q^{3}$. Hence $P$ acts regularly on $\mathcal{P}-\{x\}$. There exist $h \in P$ such that $z=y^{h}$, where for all $y, z \in \mathcal{P}-\{x\}$. Since $g \in G_{x}, h \in P$ and $P$ is a normal Sylow 3-subgroup of $G_{x}$, we have $h^{-1} g h g^{-1} \in P$. On the other hand,

$$
\mathrm{z}^{h^{-1} g h g^{-1}}=y^{g h g^{-1}}=y^{h g^{-1}}=z^{g^{-1}}=z .
$$

So $h^{-1} g h g^{-1}=1$, that is $g h=h g$. Hence $h \in C=C_{P}(g)$. We get that $C$ is transitive on $\operatorname{Fix}_{\mathcal{P}}(g)-\{x\}$. Hence $\left|F i x_{\mathcal{P}}(g)-\{x\}\right||| C \mid$. By $C \leq P$, we have $\left|F i x_{\mathcal{P}}(g)-\{x\}\right||| P \mid$. Note that $|P|=q^{3}=3^{3 f}$, so

$\mid$ Fix $_{\mathcal{P}}(g)-\{x\}|| 3^{3 f}$. Hence $\mid$ Fix $_{\mathcal{P}}(g)-\{x\} \mid \equiv 1(\bmod 2)$. It follows that $\mid$ Fix $_{\mathcal{P}}(g) \mid \equiv 0(\bmod 2)$. This means that $g$ must fix at least four points in $\mathcal{P}$.

Now, we can continue to prove our main theorem. Obviously, $\alpha$ fixes three points of $\mathcal{P}$ which are $0,1, \infty$. Then $\langle\alpha\rangle \leq G_{0,1, \infty}$. Hence $\alpha$ must fix at least five points in $\mathcal{P}$. Since $G$ acts block-transitively on $4-(v, k, 4)$ design, we can find four blocks, let $B_{1}, B_{2}, B_{3}$ and $B_{4}$, containing four points which is fixed by $\alpha$. If $\alpha$ exchange $B_{1}, B_{2}, B_{3}$ and $B_{4}$, then 2||$\langle\alpha\rangle \mid$ which is impossible. Thus $\alpha$ must fix $B_{1}, B_{2}, B_{3}$ and $B_{4}$. We have $G \cap\langle\alpha\rangle \leq G_{0 B_{1}}=G_{0 B_{2}}=G_{0 B_{3}}=G_{0 B_{4}}$. Therefore $T$ acts also flag-transitively on $4-\left(q^{3}+1, k, 4\right)$ design. We may assume $G=T$ and $|G|=q^{3}\left(q^{3}+1\right)(q-1)$.

Since $G$ acts flag-transitively on $4-\left(q^{3}+1, k, 4\right)$ design, then $G$ is point-transitive. By Lemma $1(1)$, we get

$$
\left|G_{x}\right|=\frac{|G|}{v}=\frac{q^{3}\left(q^{3}+1\right)(q-1)}{q^{3}+1}=q^{3}(q-1) .
$$

Again by Lemma 3(2) and Lemma 1(3),

$$
b=\frac{4 v(v-1)(v-2)(v-3)}{k(k-1)(k-2)(k-3)}=\frac{v\left|G_{x}\right|}{k\left|G_{x B}\right|} .
$$

Thus

$$
\left|G_{x B}\right|=\frac{(k-1)(k-2)(k-3)\left|G_{x}\right|}{4(v-1)(v-2)(v-3)}=\frac{(k-1)(k-2)(k-3) q^{3}(q-1)}{4 q^{3}\left(q^{3}-1\right)\left(q^{3}-2\right)}=\frac{(k-1)(k-2)(k-3)}{4\left(q^{2}+q+1\right)\left(q^{3}-2\right)} .
$$

By Lemma 2,

$$
4\left|G_{x B}\right|\left(q^{2}+q+1\right)\left(q^{3}-2\right)=(k-1)(k-2)(k-3) \leq(k-1) \cdot 4(v-3)=4(k-1)\left(q^{3}-2\right),
$$

Again by Corollary 1,

$$
1 \leq\left|G_{x B}\right| \leq \frac{k-1}{q^{2}+q+1} \leq \frac{2+2 q \sqrt{q}}{q^{2}+q+1}<1(q \geq 27),
$$

This is impossible.

This completes the proof the Main Theorem.

\section{References}

[1] Cameron, P.J. and Praeger, C.E. (1992) Block-Transitive t-Designs, II: Large t. In: De Clerck, F., et al., Eds., Finite 
geometry and Combinatorics, Deinze. (1993) London Math. Soc. Lecture Note Series, Vol. 191, Cambridge Univ. Press, Cambridge, 103-119.

[2] Huber, M. (2009) Flag-Transitive Steiner Designs. Birkhäuser Basel, Berlin, Boston. http://dx.doi.org/10.1007/978-3-0346-0002-6

[3] Ree, R. (1961) A Family of Simple Groups Associated with the Simple Lie Algebra of Type ( $G_{2}$ ). American Journal of Mathematics, 83, 432-462. http://dx.doi.org/10.2307/2372888

[4] Kleidman, P.B. (1988) The Maximal Subgroups of the Chevally Groups ${ }^{2} G_{2}(q)$ with $q$ Odd, the Ree Groups ${ }^{2} G_{2}(q)$, and Their Automorphism Groups. Journal of Algebra, 117, 30-71. http://dx.doi.org/10.1016/0021-8693(88)90239-6

[5] Liu, W.J., Zhou, S., Li, H. and Fan, X. (2004) Finite Linear Spaces Admitting a Ree Simple Group. European Journal of Combinatorics, 25, 311-325. http://dx.doi.org/10.1016/j.ejc.2003.10.001

[6] Dai, S.J. and Zhang, R.H. (2013) The 2-(v,13,1) Designs with Block-Transitive Automorphism. Ars Combinatoria, 110, 15-21.

[7] Dixon, J.D. and Mortimer, B. (1996) Permutation Groups. Springer Verlag, Berlin. http://dx.doi.org/10.1007/978-1-4612-0731-3

[8] Shen, H. (1990) The Theory of Combinatorial Design. Shanghai Jiaotong Univ. Press, Shanghai. 